\title{
ANÁLISE DE QUALIDADE DE DIFERENTES MARCAS DE COMPRIMIDOS DE CAPTOPRIL
}

DOMICIANO, Samyra Leandro; FARIAS, Sandra Ramos. Discentes do Curso de Farmácia do Centro Universitário de Votuporanga/Campus Centro. E-mail: samyra_leandro@hotmail.com; sandra_farias92@hotmail.com

JORDÃO, Christiane Oliveira. Docente do Curso de Farmácia do Centro Universitário de Votuporanga/ Campus Centro. Rua Pernambuco, 4196. Bairro: Centro. Votuporanga-SP. CEP: 15500-006.

E-mail: cojord@ hotmail.com

\section{RESUMO}

O controle de qualidade consiste em um conjunto de operações com o objetivo de verificar se o produto está em conformidade com as especificações da farmacopeia, que garantem a segurança, eficácia terapêutica e a qualidade deste, durante todo o prazo da validade. Para o paciente, a falta de qualidade do medicamento ocasiona sérios transtornos com o comprometimento da sua saúde. O consumo anual dos anti-hipertensivos subiu no período de janeiro de 2010 a abril de 2013, apresentando um aumento de 41,1 milhões para 119 milhões de unidades, com 16,57\% de participação nas vendas atribuídas aos medicamentos para controle da pressão arterial, em que o captopril representa 1,47\% destas. Diante desses dados, o presente trabalho propôs a quantificação do teor de comprimidos de captopril de diferentes marcas disponíveis no mercado farmacêutico. Foram realizadas análises com os comprimidos de $25 \mathrm{mg}$ de seis laboratórios diferentes, sendo três genéricos e três similares. A metodologia de análise baseou-se em método titulométrico, determinação do peso individual e médio dos comprimidos analisados, conforme apresentado na metodologia oficial. As análises foram feitas em duplicata. Os dados obtidos foram estatisticamente avaliados determinando o coeficiente de variação e desvio padrão. Apesar das marcas analisadas apresentarem diferenças estatísticas, pode-se afirmar que, dentre os testes realizados, todas as amostras estavam dentro dos padrões estabelecidos.

Palavras-chave: Controle de qualidade; Análise; Captopril.

\section{QUALITY ANALYSIS OF DIFFERENT BRANDS OF CAPTOPRIL TABLETS}

\begin{abstract}
Quality control consists of a set of operations in order to verify if the product conforms to the specifications of the pharmacopoeia, to assure its safety, its therapeutic efficacy and its quality, throughout the validity period. For the patient, the lack of quality of the product causes serious trouble with health risks. The annual consumption of antihypertensive drugs rose from January 2010 to April 2013, an increase of 41.1 million to 119 million units, with $16.57 \%$ share of sales attributable to drugs to control blood pressure in which captopril represents $1.47 \%$. Considering these data, the present study aimed at quantifying the content of captopril tablets of different brands available in the pharmaceutical market. Analyses were performed with $25 \mathrm{mg}$ tablets of six different laboratories, being three similar and three generic ones. The analysis methodology was based on titrimetric method, determining the individual and mean weight of the tablets analyzed, as shown in the official methodology. Duplicate analyzes were performed. The data were statistically evaluated by determining the coefficient of variation and standard deviation. Despite the analyzed brands present statistical differences, it is possible to confirm that the tests of all samples were within the established standards.
\end{abstract}


KeYwords: Quality Control, Analysis, Captopril.

\section{INTRODUÇÃo}

A qualidade dos dados analíticos representa um fator chave no sucesso de um programa de desenvolvimento de uma droga, e o processo de produção e validação dos métodos analíticos tem impacto direto na qualidade desses dados (MORETTO; SHIB, 2000).

O controle de qualidade consiste em um conjunto de operações com o objetivo de verificar se o produto está em conformidade com as especificações da farmacopeia. A não conformidade representa um somatório de atribulações para a empresa que pode resultar, além dos prejuízos decorrentes do retrabalho, na perda de credibilidade e até na cassação da licença de funcionamento e do registro do produto (PEIXOTO et al., 2005).

A Agência Nacional de Vigilância Sanitária (ANVISA, 2006) determinou a interdição cautelar em todo o país de lotes do medicamento similar antihipertensivo captopril. Análises do Instituto Nacional de Controle de Qualidade em Saúde constataram que o teor de princípio ativo estava acima do permitido.

No ano de 2006, a ANVISA determinou a interdição cautelar do anti-hipertensivo propranolol. Laudos do Instituto Nacional de Controle de Qualidade em Saúde - INCQS detectaram desvios de qualidade nos ensaios de teor do princípio ativo e de rótulo (uso incorreto da denominação do princípio ativo na embalagem primária do medicamento).

A cada ano morrem 7,6 milhões de pessoas em todo o mundo devido à hipertensão, segundo dados do Boletim Global de Doenças Relacionadas à Hipertensão. A hipertensão arterial é responsável, segundo o documento, por $54 \%$ de todos os casos de acidente vascular cerebral - AVC e $47 \%$ dos casos de infarto, fatais e não fatais, em todo o mundo. $\mathrm{Na}$ última década, a hipertensão fez mais de 70 milhões de vítimas fatais. Cerca de $80 \%$ dessas mortes ocorrem em países em desenvolvimento como o Brasil, e mais da metade das vítimas têm entre 45 e 69 anos (LAWES, VANDER HOOM \& RODGERS, 2008).
A hipertensão constitui-se um problema de saúde pública, pois atinge 17 milhões de brasileiros, dos quais $75 \%$ recorrem à Estratégia de Saúde à Família - ESF. Para o tratamento farmacológico dessa patologia existem diversas classes de medicamentos, entretanto, os inibidores orais da enzima conversora da angiotensina - ECA são frequentemente prescritos pelos médicos, devido à sua comprovada eficácia (TEIXEIRA et al., 2010)

De acordo como Ministério da Saúde (2007), para o seu tratamento foram distribuídos, em 2005, 5,6 bilhões de medicamentos para hipertensos, crescimento de $65,24 \%$ em relação a 2004. Cerca de 7,7 milhões de brasileiros estão cadastrados na ESF e já recebem os medicamentos gratuitamente.

Os medicamentos anti-hipertensivos são medicamentos utilizados com ação direta nos órgãos, melhorando sua eficiência e produzindo efeito hipotensor, que podem ser classificados de acordo com seus locais ou mecanismos de ação, como: I) diuréticos; II) agentes simpatolíticos; III) vasodilatadores; IV) bloqueadores dos canais de cálcio; V) inibidores da ECA e antagonistas do receptor da angiotensina II (VALENTIM; SOMMER; MATIOLI, 2004).

Os inibidores da ECA são substâncias que diminuem a pressão arterial por bloquearem a geração de angiotensina II, um dos mais potentes vasocontritores naturais, por meio da enzima conversora de angiotensina I (BARBOZA, 2012).

O captopril foi descoberto como o primeiro fármaco anti-hipertensivo inibidor da ECA, combase em estudos que evidenciaram os efeitos hipotensores do veneno da jararaca, da espécie Bothrops jararaca (BARREIRO; FRAGA, 2001). No controle da pressão arterial, a terapêutica medicamentosa tem-se apresentado eficaz, colaborando para a diminuição de eventos cardiovasculares (RIBAS \& GUIMARÃES, 2006).

Segundo a Farmacopeia Brasileira V (2010) 2. Edição, o captopril constitui-se em um pó cristalino, branco ou quase branco, solúvel em água, facilmente solúvel em metanol e cloreto de metileno, solúvel em soluções diluídas de hidróxidos alcalinos, com faixa de fusão de $105^{\circ} \mathrm{C}$ a $108^{\circ} \mathrm{C}$. 
Estudos relatam diversos métodos para quantificação dessa matéria-prima e em formulações farmacêuticas convencionais, como cromatografia líquida de alta eficiência-CLAE, volumetria, métodos fluorimétricos, colorimétricos, espectroscopia Raman, eletroforese e espectroscopia no ultravioleta. Além dessas técnicas, a espectroscopia no ultravioleta também é indicada para sua quantificação durante o ensaio de dissolução (SHAMA, AMIN, OMARA 2006; THE UNITED STATES PHARMACOPEIA, 2000).

Dentre os métodos citados acima, a titulação volumétrica é uma técnica bastante utilizada, por permitir a obtenção de resultados precisos e exatos, desde que realizados por pessoas treinadas, com baixo custo, rapidez de resposta, pouco consumo e simplicidade/disponibilidade da maioria dos reagentes (LEITE, 2004).

Diante desses dados, o presente trabalho propôs a quantificação do captopril em comprimidos, de diferentes marcas disponíveis no mercado farmacêutico, para verificar se estão em conformidade com o estabelecido em seus rótulos e bulas.

\section{Materiais e Métodos}

Para o ensaio de peso médio foram utilizados 12 comprimidos de cada amostra, pesados individualmente de acordo com a Farmacopeia Brasileira V (2010). Pode-se tolerar não mais que duas unidades fora dos limites especificados. Nenhuma amostra pode estar acima ou abaixo do dobro das porcentagens indicadas, conforme tabela presente na Farmacopeia Brasileira. Os dados obtidos também foram estatisticamente avaliados com relação ao coeficiente de variação e desvio padrão.

O procedimento analítico utilizado para avaliação das amostras foi adaptado da monografia oficial descrita na USP XXIV (2000), referente à matériaprima do captopril.

Foi preparada uma solução de iodato de potássio 0,1N em água destilada e padronizada com solução de permanganato de potássio $0,1 \mathrm{~N} \mathrm{~S}$.V.

Foram pesados 12 comprimidos para realização do tesde em cada amostra, equivalente a $300 \mathrm{~g}$ de captopril. Cada tomada de ensaio foi transferida para um erlenmeyer de $250 \mathrm{ml}$, ao qual foram adicionados $1 \mathrm{~g}$ de iodeto de potássio, $100 \mathrm{ml}$ de água destilada, $10 \mathrm{ml}$ de ácido sulfúrico 3,6 N e $2 \mathrm{~mL}$ de goma de amido $1 \%$. Realizou-se a titulação utilizando iodato de potássio $0,1 \mathrm{~N} \mathrm{S.V}$., até coloração azul persistente por 30 segundos. Um branco foi preparado nas mesmas condições que a amostra, com exceção do captopril. Todas as análises foram feitas em duplicata.

O cálculo da concentração de princípio ativo nos comprimidos foi efetuado considerando que cada $\mathrm{mL}$ de iodato de potássio $0,1 \mathrm{~N}$ equivale a $21,73 \mathrm{mg}$ de captopril.

Os dados obtidos foram estatisticamente avaliados com relação ao coeficiente de variação e desvio padrão.

Para os testes de peso individual e teor dos comprimidos, foram utilizados aproximadamente 150 comprimidos de captopril contendo $25 \mathrm{mg}$, de seis laboratórios diferentes, sendo três genéricos e três similares, adquiridos em farmácias privadas.

\section{Resultados e Discussão}

$\mathrm{Na}$ Tabela 1 estão relacionados os dados obtidos na análise de teor para as diferentes marcas de captopril.

O ensaio de determinação do teor destina-se à avaliação da quantidade de substância ativa contida no medicamento. Este serve para identificar se o paciente está ingerindo uma dose correta de substância ativa, não sendo subdose ou sobredose. Com um fármaco potente, tal como a digitoxina, em que a concentração terapêutica e a concentração tóxica são próximas, ou mesmo sobreponíveis, ultrapassar a dose administrada ao doente não só é indesejável, como perigoso (BANKER; ANDERSON, 2001).

Com os resultados das análises realizadas foi possível inferir que o teor de ativos em medicamentos genéricos e similares não difere significativamente. Os teores de captopril encontrados nos comprimidos analisados variaram entre $22,83 \mathrm{mg}$ - 24,99 mg. A USP XXIV estabelece que esses comprimidos tenham no mínimo $90,0 \%$, o que 
corresponde a $22,5 \mathrm{mg}$ e, no máximo, $110,0 \%$, que corresponde a 27,5 mg da quantidade declarada de substância ativa. Sendo assim, no presente trabalho pode-se constatar que todas as amostras estavam em conformidade quanto ao teor do fármaco.

O desvio padrão e coeficiente de variação das análises das amostras de genéricos 1 e 2 e similares $2 \mathrm{e}$ 3 foramigual a 0 , isso quer dizer que não houve variação das amostras em relação à média; já as amostras genérico 3 e similar 1 tiveram variação em seus resultados, apresentando valores de desvio padrão e coeficiente de variação maior que zero (Tabela 1).

Tabela 1 - Teor de Princípio Ativo encontrados nos comprimidos de captopril de cada amostra.

\begin{tabular}{ccccc}
\hline Amostras & Teor $(\mathbf{m g})$ & Referência $(\mathbf{m g})$ & Desvio Padrão & $\begin{array}{c}\text { Coeficiente de } \\
\text { Variação (\%) }\end{array}$ \\
\hline Genérico 1 & 23,90 & 22,5 a 27,5 & 0 & 0 \\
Genérico 2 & 22,82 & 22,5 a 27,5 & 0 & 0 \\
Genérico 3 & 24,99 & 22,5 a 27,5 & 1,52 & 6,08 \\
Similar 1 & 23,36 & 22,5 a 27,5 & 3,64 & 3,64 \\
Similar 2 & 24,99 & 22,5 a 27,5 & 0 & 0 \\
Similar 3 & 23,90 & 22,5 a 27,5 & 0 & 0 \\
\hline
\end{tabular}

Fonte: Dados da Pesquisa.

Durante a realização das análises, além do teor do princípio ativo foi possível analisar o peso médio das diferentes amostras de comprimidos. De acordo com a Farmacopeia Brasileira V (2010), em relação ao peso médio dos comprimidos não revestidos, que pesam entre 80 e $250 \mathrm{mg}$, quando for $80 \mathrm{mg}$ ou menos a variação fica entre $\pm 10,0 \%$, mais de $80 \mathrm{mg}$ e menos que $250 \mathrm{mg} \pm 7,5 \%$ e $250 \mathrm{mg}$ ou mais $\pm 5,0 \%$, não são permitidos mais que duas unidades fora do limite, nenhuma pode estar o dobro do peso acima ou abaixo.

Foram também calculados os valores máximos e mínimos permitidos, de acordo com a Farmacopeia Brasileira V (2010) para cada amostra. As variações de peso permitidas estão demonstradas na Tabela 2. 
Tabela 2 - Valores Mínimos e Máximos permitidos para cada amostra.

\begin{tabular}{ccc}
\hline & Peso Mínimo Permitido & Peso Máximo Permitido \\
Genérico 1 & $0,1581 \mathrm{~g}$ & $0,1837 \mathrm{~g}$ \\
Genérico 2 & $0,1508 \mathrm{~g}$ & $0,1752 \mathrm{~g}$ \\
Genérico 3 & $0,0751 \mathrm{~g}$ & $0,1684 \mathrm{~g}$ \\
Similar 1 & $0,0944 \mathrm{~g}$ & $0,1098 \mathrm{~g}$ \\
Similar 2 & $0,0928 \mathrm{~g}$ & $0,1078 \mathrm{~g}$ \\
Similar 3 & $0,1494 \mathrm{~g}$ & $0,1736 \mathrm{~g}$ \\
\hline
\end{tabular}

Fonte: Dados da Pesquisa.

De acordo com a Tabela 3 é possível verificar que cada uma das unidades avaliadas estava dentro dos limites determinadosto para o peso médio. 
Tabela 3 - Peso Unitário e Médio dos comprimidos analisados.

\begin{tabular}{|c|c|c|c|c|c|c|}
\hline Unidade & $\begin{array}{c}\text { Genérico } 1 \\
\text { (g) }\end{array}$ & $\begin{array}{c}\text { Genérico } 2 \\
\text { (g) }\end{array}$ & $\begin{array}{c}\text { Genérico } 3 \\
\text { (g) }\end{array}$ & $\begin{array}{c}\text { Similar } 1 \\
(\mathrm{~g})\end{array}$ & $\begin{array}{c}\text { Similar } 2 \\
\text { (g) }\end{array}$ & $\begin{array}{c}\text { Similar } \\
\text { (g) }\end{array}$ \\
\hline 1 & 0,164 & 0,163 & 0,078 & 0,101 & 0,098 & 0,163 \\
\hline 2 & 0,174 & 0,167 & 0,078 & 0,101 & 0,103 & 0,163 \\
\hline 3 & 0,174 & 0,169 & 0,080 & 0,102 & 0,101 & 0,164 \\
\hline 4 & 0,173 & 0,156 & 0,084 & 0,102 & 0,101 & 0,160 \\
\hline 5 & 0,175 & 0,165 & 0,081 & 0,105 & 0,100 & 0,163 \\
\hline 6 & 0,166 & 0,163 & 0,081 & 0,102 & 0,099 & 0,160 \\
\hline 7 & 0,173 & 0,157 & 0,084 & 0,102 & 0,099 & 0,160 \\
\hline 8 & 0,169 & 0,170 & 0,079 & 0,101 & 0,101 & 0,162 \\
\hline 9 & 0,169 & 0,168 & 0,081 & 0,101 & 0,100 & 0,161 \\
\hline 10 & 0,170 & 0,158 & 0,082 & 0,102 & 0,101 & 0,162 \\
\hline 11 & 0,171 & 0,154 & 0,081 & 0,101 & 0,101 & 0,160 \\
\hline 12 & 0,173 & 0,166 & 0,086 & 0,105 & 0,100 & 0,161 \\
\hline Peso Médio & 0,1709 & 0,1630 & 0,0812 & 0,1021 & 0,1003 & 0,1615 \\
\hline Desvio Padrão & 0,0140 & 0,0017 & 0,0025 & 0,0014 & 0,0099 & 0,0056 \\
\hline Coeficiente de & $8,19 \%$ & $1,04 \%$ & $3,08 \%$ & $1,37 \%$ & $9,87 \%$ & $3,47 \%$ \\
\hline Variação & & & & & & \\
\hline
\end{tabular}

Fonte: Dados da Pesquisa.

O desvio padrão é capaz de identificar o "erro" em junto à média aritmética, informando o quão "confiável" um conjunto de dados, caso quiséssemos substituir um dos valores coletados pela média aritmética. Aparece é esse valor. Conforme o Formulário Nacional da Farmacopeia Brasileira (2012), esse Desvio padrão 
relativo - DPR calculado não deve ser maior que $4 \%$.

$\mathrm{O}$ coeficiente de variação analisa a dispersão em termos relativos, ele é dado em \%. Quanto menor for o valor do coeficiente de variação, mais homogêneos serão os dados, ou seja, menor será a dispersão em torno da média. De uma forma geral, se o Coeficiente de Variação for menor ou igual a $15 \%$ ? baixa dispersão: dados homogêneos; entre 15 e $30 \%$ ? média dispersão; maior que $30 \%$ ? alta dispersão: dados heterogêneos (RIGONATTO, 2012).

Com relação ao coeficiente de variação dos resultados obtidos no presente trabalho, pode-se verificar que, tanto para o teor de captopril quanto para o peso médio, houve baixa dispersão, ou seja, dados foram homogêneos, estando abaixo de $15 \%$.

Valentim, Sommer e Matioli (2004), através de uma análise realizada no município de Maringá, buscaram a validação de método analítico para quantificação de comprimidos de captopril. Foi utilizado o equivalente a $300 \mathrm{mg}$ de captopril, onde os resultados de quatro amostras apresentaram valores acima de $105 \%$, que, mesmo assim, estão dentro da faixa de especificação determinada pela metodologia seguida - USP XXIV (90\% a 110\%). A precisão foi considerada aceitável, pois apresentaram um coeficiente de variação menor que $15 \%$. No presente trabalho, todos os resultados do teor de captopril também estiveram dentro da faixa de especificação determinada pela metodologia, mas nenhuma amostra atingiu um teor igual ou maior que $100 \%$ e o coeficiente de variação também apresentou resultados positivos apresentando valores abaixo de $15 \%$, o que mostra que os dados foram homogêneos.

De acordo com Becktold (2008), em uma pesquisa realizada em Blumenau, em que foi avaliada a qualidade de comprimidos de captopril genérico, similar e referência, os resultados obtidos na análise de teor foram para a Amostra A 98\%, Amostra B 99,9\%, Amostra C 99,4\% e Amostra D 100\%, estando assim todas em conformidade com os parâmetros requeridos. Na avaliação do peso médio as amostra A, B e D foram aprovadas, sendo a amostra $\mathrm{C}$ reprovada.
Abido \& Knorst (2014) buscaram a Avaliação da qualidade de comprimidos de captopril comercializados no Brasil, através de uma pesquisa realizada em Passo Fundo-RS, em que realizaram testes de peso médio, teor de comprimidos, entre outros, e obtiveram resultados que demonstram que todos os produtos avaliados estão de acordo com a especificação de no mínimo $90 \%$ e no máximo $110 \%$ da quantidade declarada de captopril; em relação ao peso médio todas as amostras também atenderam às especificações.

Já na presente pesquisa, todas as amostras utilizadas foram aprovadas, tanto no teste do teor de captopril, quanto no teste de peso médio, apresentando também um valor de Desvio Padrão e Coeficiente de Variação dentro de padrões aceitáveis.

\section{Conclusão}

A análise de medicamentos é realizada rotineiramente pelos laboratórios da indústria farmacêutica, por ser crucial para garantir a qualidade do produto e para maior segurança aos seus usuários. Dessa forma, este trabalho propõe contribuir para avaliar a qualidade e a segurança de comprimidos de captopril, bem como para assegurar sua eficácia no tratamento da hipertensão.

A partir dos resultados obtidos para os parâmetros investigados neste trabalho, apesar das marcas analisadas apresentarem diferenças estatísticas quando comparados aos valores obtidos dos diferentes testes, pode-se concluir que, dentre os testes realizados, todas as amostras estiveram dentro dos padrões estabelecidos, tanto nas análises do teor, quanto do peso médio, apresentando também um Desvio Padrão e Coeficiente de Variação positivos.

\section{Agradecimentos}

As alunas agradecem ao Laboratório de Análises do Centro Universitário de Votuporanga-SP/Campus Centro, pela disponibilidade para realização das análises para o presente trabalho, ao responsável pelo Laboratório Rafael Ramires pela colaboração dada ao nosso trabalho. 


\section{REFERÊNCIAS}

ANVISA. Anvisa interdita lotes de anti-

hipertensivos. Disponível em: http://

www.anvisa.gov.br/divulga/noticias/2006/

120106.htm. Acesso em: 04 out. 2014.

ABIDO \& KNORST. Avaliação da qualidade de comprimidos de captopril comercializados no

Brasil. Passo Fundo, 2014.

BARBOZA. Anti-hipertensivos, 2012. Disponível em: http://pt.slideshare.net/RenatoSantos40/aulaantihipertensivos. Acesso:08 Dez. 2014.

BANKER, G. S.; ANDERSON, N.R. Comprimidos. In: LACHMAN, L.; LIEBERNAN, H. A.; KANIG, J. L. Teoria e Prática na Indústria Farmacêutica. 7 ed. Lisboa: Fundação Calouste Gulbenkian, 2001. p. 509-526.

BARREIRO; J,; FRAGA, C. A. M. Planejamento racional baseado no mecanismo de ação: fármacos inteligentes. In: Química medicinal: as bases moleculares da ação dos fármacos. Porto Alegre: Artmed, 2001. P. 83-124.

BECKTOLD. Análise comparativa da qualidade de comprimidos de Captopril: genérico, similar e de referência, 2008.

FARMACOPEIABRASILEIRA. Agência Nacional de Vigilãncia Sanitária. Brasília: Anvisa, 2010. v.1

FARMACOPEIABRASILEIRA/ Agência Nacional de Vigilância Sanitária. Brasília: Anvisa, 2010. v.2

FORMULÁRIO NACIONAL DA FARMACOPEIABRASILEIRA: desvio padrão relativo (DPR), 2.ed. 2012.

LAWES CM., VANDER HOOM S \& RODGERS
A. Global burden of blood-pressure-related disease, 2001. International Society oh Hypertension.

Lancet, v.371, n.9623, p, 1513-1518, 2008.

LEITE, F. AFantástica Titrimetria-Titulação

Volumétrica, Revista Analytica. Agos./Set., 2004.

MAGALHÃES, L. B. N. Anti-hipertensivos. In: SILVA, P. (Ed.). Farmacologia. Rio de Janeiro: Guanabara Koogan, 1998. cap. 66, p. 647-657.

MINISTÉRIO DA SAUDE: Relação nacional de medicamentos essenciais: Rename. 7 e.d. Brasília: Serie B. Textos Básicos de Saúde. 250 p. Disponível em: http://portal.saude.gov.br/portal/ arquivos/pdf/rena me2007final.pdf. Acesso em: 18 Jan. 2014.

MINISTÉRIO DA SAÚDE. Hipertensão Arterial. Disponível em http://portalsaude.saude.gov.br/ Acesso em: 20 Jan. 2014.

MORETTO, L. D.; SHIB, M. A era da validação. Rev. Pharmaceut. Technol., São Paulo, v. 4, n. 4, p. 44-48, 2000.

PEIXOTO, M. M.; SANTOS, A. F. JR.; SANTOS, C. A. A.; CAETITÉ, E. JR. Avaliação da Qualidade de Comprimidos de Captopril Dispensados em Feira de Santana-BA. Infarma, v.16, n. 13-14, pg. 69-73, 2005.

RIBAS, L. F. O.; GUIMARÃES, V. M. Avaliação da compreensão dos pacientes hipertensos a respeito da hipertensão arterial e seu tratamento versus controle pressórico. Revista Brasileira de Medicina de Família e Comunidade, v. 1, n. 4, p. 152-164, 2006.

RIGONATTO. Coeficiente de Variação: Mundo Educação. Disponivel em: http:// www.mundoeducacao.com/matematica/ coeficiente-variacao.htm\#comentarios. Acesso 
em: 0609 set. 2014.

SHAMA S.A.; AMIN A.E.; OMARA H., Colorimetric microdetermination of captopril in pure form and in pharmaceutical formulations, J. Quant. Spectrosc. Radiat. Transfer, v.102, p.261 2006.

TEIXEIRA, C.M.; SANTOS, P.M.; SAKANE, K.K.; UEHARA, M.; CARDOSO, M.A.G. Análise qualitativa de captopril em amostras de medicamentos disponibilizados em postos públicos utilizando espectroscopia ft-ir, 2010. In:

ENCONTRO LATINO-AMERICANO DE PÓS-
GRADUAÇÃO.10, Universidade do Vale do Paraíba, 2010.

THE UNITED STATES PHARMACOPEIA, XXIV, United States Pharmacopeial Convention, 2000.

VALENTIN; SOMMER; MATIOLI. Validação de Métodos Analíticos na Quantificação de comprimidos de Captopril:Comparação de metodologias para um programa de garantia de qualidade. Acta Scientarum, v. 26, n. 2, 2004. 